\title{
A PORTABLE HOLOGRAPHIC ANTENNA MEASUREMENT SYSTEM
}

\author{
DAVID B. SHAFFER and JOHN M. GIPSON
}

Interferometrics Inc., 8150 Leesburg Pike, Vienna, Virginia 22182

\begin{abstract}
Interferometrics has developed a portable holographic antenna measurement system. The system is comprised of a small interferometer which uses satellite signals to measure the complex beam pattern of the antenna under test. A PC controls all aspects of data collection and analysis. The system produces maps of surface profile errors and the illumination power in reflector antennas.
\end{abstract}

The portable holographic antenna measurement system is a commercial application of radio astronomy interferometry techniques and hardware.

Radio holography makes use of the Fourier-transform relation between the currents in the aperture of an antenna and the beam pattern of the antenna. If the complex beam pattern (real and imaginary) is measured, the Fourier transform gives the complex currents in the antenna aperture. The phase of the current indicates areas of advance or retardation of the aperture wavefront, which are caused by high or low spots on the reflecting surface(s) of the antenna. Thus, the phase map shows the surface height errors. The accuracy of the surface measurement is generally better than 0.01 wavelength $(0.25$ millimeters or better for measurements at $12 \mathrm{GHz}$ ). The amplitude of the aperture current shows the aperture illumination which reveals feed taper, shadowing by feed legs, subreflectors or equipment at the focus, and areas of low reflectivity.

The holographic antenna measurement system measures the complex beam pattern with a short baseline interferometer comprised of the antenna under test and a small reference antenna. Baselines of 50 to 100 meters are typical. The interferometer uses a multi-lag digital correlator, similar to those used in most radio astronomy interferometers. The correlator dumps data to the PC many times per second. The PC can integrates as many of the data dumps as desired.

The signal sources for our measurements are satellites. Most of our measurements have been made with geostationary satellites transmitting at $4,7.5$, or $12 \mathrm{GHz}$. We measured the Millstone antenna at $1.23 \mathrm{GHz}$ while the antenna tracked GPS satellites. The satellite signals are so strong that very small reference antennas ( 1 to 2 meter diameter) can be used. For the Millstone measurements, the reference antenna was just a horn mounted on the big antenna. We do not demodulate the satellite signals, but process them just like cosmic sources, in typical bandwidths of 0.1 to $1 \mathrm{MHz}$. 
A '386 class PC controls the complete holography system. It determines where the antenna is pointed, controls the operation of the correlator, performs the FFT on the data, and analyzes the aperture maps. Figure 1 shows a block diagram of the system.
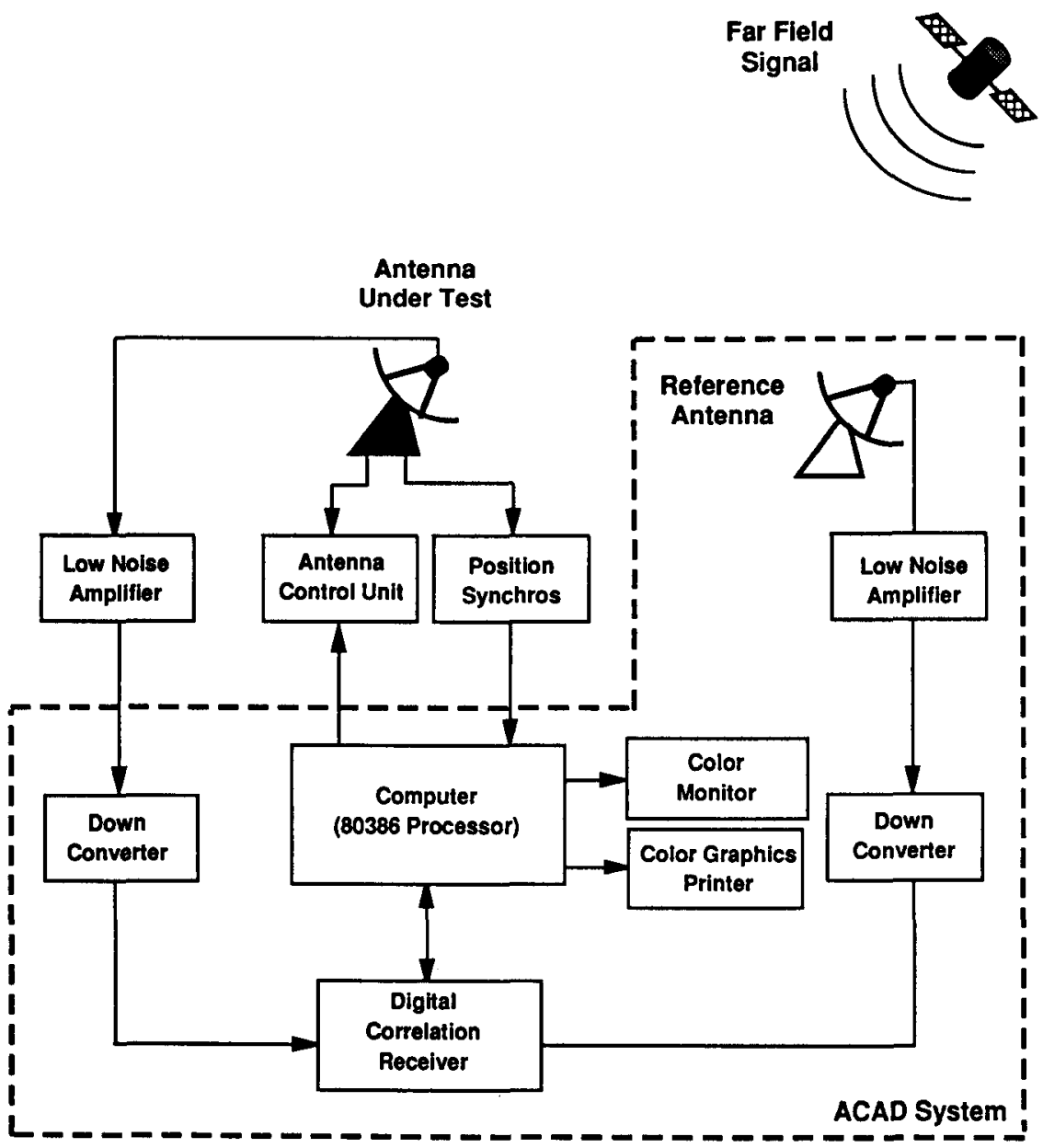

Fig. 1. Block diagram of the holography system. The components inside the dashed outline make up the Interferometrics system. 
The beam pattern is measured in a grid with angular spacings of slightly less than $\frac{\lambda}{D}$ (the Nyquist spacing). By slightly oversampling the beam pattern, the image of the aperture stays within the FFT map boundary. At first thought, it seems desirable to point the antenna explicitly at the grid points. However, the time required for the antenna to move and settle down is much greater than the time actually required to make a measurement. (A fraction of a second of data is usually adequate, whereas moving the antenna may take several seconds.) For a large grid, this overhead is intolerable, and we make most of our maps by taking data "on the fly." In this mode, the antenna is pointed to the desired azimuth and then scanned in elevation. When the antenna is close to a grid point (typically within 5 or 10grid spacing), the PC takes data from the correlator. The antenna under test returns periodically to the bore site position for phase and amplitude calibration. The total power in the reference antenna is monitored continuously, and the correlation coefficients are normalized for any variation in the signal level from the satellite.

Implementing a pointing interface for an arbitrary antenna has proven to be a time-consuming and therefore expensive task. Some antennas are not even amenable to computer control. They have only rudimentary pointing systems, with joystick controls and direct display of pointing coordinates. Our goal is to measure an antenna quickly and with minimum downtime. Therefore, we have implemented techniques which are as independent of the antenna pointing system as possible. In general, we control the pointing of the antenna with its "native" pointing system, and the PC receives only pointing feedback. For some antennas, we can connect the PC directly to the encoders. For others, we use a tiltmeter to determine relative elevations. Setting the azimuth by hand and then scanning in elevation works very well for the data-on-the-fly mode.

Once the data have been collected, the PC does a fast Fourier transform to get the aperture map. A 128 by 128 transform takes only a few seconds on a $25 \mathrm{MHz}$ PC. The aperture map can be analyzed for various parameters. The optimum feed location can be determined, along with recommended settings for all the panels in an antenna. The rms surface error, efficiency, and gain of the antenna can be calculated, both for the original data and for the expected improvements if the focus and panels are adjusted. Similarly, before and after beam patterns can be compared. The PC can provide contour plots and color renditions of the antenna maps.

Figures 2a and 2b show holography maps for a 38-foot antenna which has a monopod cassegrain configuration. The illumination of this antenna is very uniform, leading to the maximum possible gain. Only the central shadowing from the monopod is seen. The surface map shows features which are clearly related to the individual panels which make up the surface. 

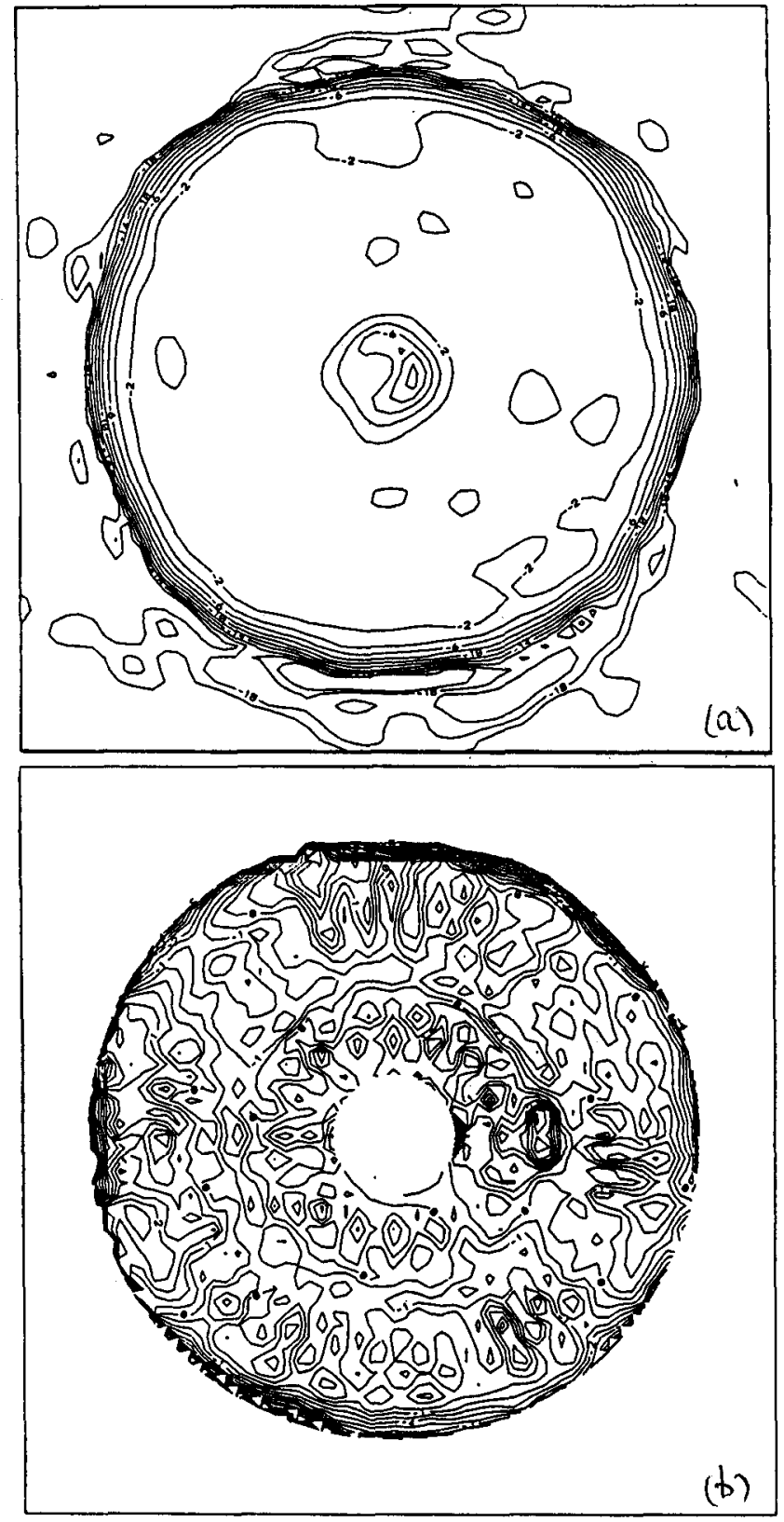

Fig. 2. a) Aperture illumination function of a 38-foot antenna with a monopod cassegrain feed. Contour interval is $2 \mathrm{~dB}$. The lowest contour is $-20 \mathrm{~dB}$ with respect to the maximum power in the aperture. b) Contour map of surface errors for the 38-foot antenna. Contour interval is $0.5 \mathrm{~mm}$. 